9. Qian, S., et al. 2002. Neither agouti-related protein nor neuropeptide $\mathrm{Y}$ is critically required for the regulation of energy homeostasis in mice. Mol. Cell. Biol. 22:5027-5035.

10. Gropp, E., et al. 2005. Agouti-related peptideexpressing neurons are mandatory for feeding. Nat. Neurosci. 8:1289-1291.

11. Luquet, S., Perez, F.A., Hnasko, T.S., and Palmiter, R.D. 2005. NPY/AgRP neurons are essential for feeding in adult mice but can be ablated in neonates. Science. 310:683-685.

12. Wortley, K.E., et al. 2005. Absence of ghrelin protects against early-onset obesity. J. Clin. Invest 115:3573-3578. doi:10.1172/JCI26003.

13. Zigman, J.M., et al. 2005. Mice lacking ghrelin receptors resist the development of diet-induced obesity. J. Clin. Invest. 115:3564-3572. doi:10.1172/ JCI26002.

14. Grove, K.L., Allen, S., Grayson, B.E., and Smith, M.S. 2003. Postnatal development of the hypothalamic neuropeptide Y system. Neuroscience. 116:393-406.

15. Grove, K.L., and Smith, M.S. 2003. Ontogeny of the hypothalamic neuropeptide Y system. Physiol. Behav. 79:47-63.

16. Bouret, S.G., Draper, S.J., and Simerly, R.B. 2004. Formation of projection pathways from the arcuate nucleus of the hypothalamus to hypothalamic regions implicated in the neural control of feeding behavior in mice. J. Neurosci. 24:2797-2805.

17. Bouret, S.G., Draper, S.J., and Simerly, R.B. 2004. Trophic action of leptin on hypothalamic neurons that regulate feeding. Science. 304:108-110.

18. Ahima, R.S., and Hileman, S.M. 2000. Postnatal regulation of hypothalamic neuropeptide expression by leptin: implications for energy balance and body weight regulation. Regul. Pept. 92:1-7.

19. Hayashida, T., et al. 2002. Ghrelin in neonatal rats: distribution in stomach and its possible role. J. Endocrinol. 173:239-245.

20. Pinto, S., et al. 2003. Rapid re-wiring of arcuate nucleus feeding circuits by leptin. Science. 304:110-115.

21. Hollopeter, G., Erickson, J.C., and Palmiter, R.D. 1998. Role of neuropeptide Y in diet-, chemicaland genetic-induced obesity of mice. Int. J. Obesity. 22:506-512.

22. Shimada, M., Tritos, N.A., Lowell, B.B., Flier, J.S., and Maratos-Flier, E. 1998. Mice lacking melaninconcentrating hormone are hypophagic and lean. Nature. 396:670-674

23. Holst, B., Brandt, E., Bach, A., Heding, A., and Schwartz, T.W. 2005. Nonpeptide and peptide growth hormone secretagogues act both as ghrelin receptor agonist and as positive or negative allosteric modulators of ghrelin signaling. Mol. Endocrinol. 19:2400-2411.

24. Holst, B., et al. 2004. Common structural basis for constitutive activity of the ghrelin receptor family. J. Biol. Chem. 279:53806-53817.

25. Holst, B., Cygankiewicz, A., Jensen, T.H., Ankersen, M., and Schwartz, T.W. 2003. High constitutive signaling of the ghrelin receptor - identification of a potent inverse agonist. Mol. Endocrinol. 17:2201-2210.

26. Lall, S., et al. 2004. Physiological studies of transgenic mice overexpressing growth hormone $(\mathrm{GH})$ secretagogue receptor $1 \mathrm{~A}$ in $\mathrm{GH}$-releasing hormone neurons. Endocrinology. 145:1602-1611.

27. Sar, M., Sahu, A., Crowley, W.R., and Kalra, S.P. 1990. Localization of neuropeptide-Y immunoreactivity in estradiol-concentrating cells in the hypothalamus. Endocrinology. 127:2752-2756.

28. Eckel, L.A. 2004. Estradiol: a rhythmic, inhibitory, indirect control of meal size. Physiol. Behav. 82:35-41

29. Nunez, A.A., and Grundman, M. 1982. Testosterone affects food intake and body weight of weanling male rats. Pharmacol. Biochem. Behav. 16:933-936.

30. Nunez, A.A. 1982. Dose-dependent effects of testosterone on feeding and body weight in male rats. Behav. Neural. Biol. 34:445-449.

31. Sohn, E.H., Wolden-Hanson, T., and Matsumoto, A.M. 2002. Testosterone (T)-induced changes in arcuate nucleus cocaine-amphetamine-regulated transcript and NPY mRNA are attenuated in old compared to young male brown Norway rats: contribution of $\mathrm{T}$ to age-related changes in cocaineamphetamine-regulated transcript and NPY gene expression. Endocrinology. 143:954-963.

32. Kobelt, P., et al. 2005. Anti-ghrelin SPIEGELMER NOX-B11 inhibits neurostimulatory and orexigenic effects of peripheral ghrelin in rats. Gut. doi:10.1136/gut.2004.061010.

33. Helmling, S., et al. 2004. Inhibition of ghrelin action in vitro and in vivo by an RNA-Spiegelmer. Proc. Natl. Acad. Sci. U. S. A. 101:13174-13179.

34. Beck, B., Richy, S., and Stricker-Krongrad, A. 2004. Feeding response to ghrelin agonist and antagonist in lean and obese Zucker rats. Life Sci. 76:473-478.

\title{
Expanding the immunotherapeutic potential of minor histocompatibility antigens
}

\author{
Eric Spierings and Els Goulmy
}

Department of Immunohematology and Blood Transfusion, Leiden University Medical Center, Leiden, The Netherlands.

\begin{abstract}
Minor histocompatibility antigens (mHAgs) selectively expressed by cells or cell subsets of the hematopoietic system are targets of the $T$ cell-mediated graft-versus-leukemia response that develops following allogeneic hematopoietic stem cell transplantation (HSCT) for the treatment of hematological malignancies. This observation has served as the rationale for utilizing mHAg-specific immunotherapy for the treatment of particular patients. However, at present, only a select and small number of patients could potentially benefit from mHAg-based immunotherapy. A report from de Rijke et al. in this issue of the JCI describes a new hematopoietic lineage-specific HLA-B7-restricted mHAg associated with remission of chronic myeloid leukemia (see the related article beginning on page 3506). This result represents another example of an mHAg-mediated graft-versus-leukemia response, thereby expanding the number of patients eligible for $\mathrm{mHAg}$-based immunotherapy in the setting of HSCT.
\end{abstract}

Nonstandard abbreviations used: GVHD, graft-versus-host disease; GVL, graft-versus-leukemia; HSCT, hematopoietic stem cell transplantation; LRH-1, lymphoid-restricted histocompatibility antigen-1; mHAg, minor histocompatibility antigen.

Conflict of interest: The authors have declared that no conflict of interest exists.

Citation for this article: J. Clin. Invest. 115:3397-3400 (2005). doi:10.1172/JCI27094.

\section{Characteristics of minor histocompatibility antigens applicable for immunotherapy}

Minor histocompatibility antigens (mHAgs) were originally defined in mice by characterization of in vivo rejection responses to skin grafts and tumors exchanged between mice of different inbred strains $(1,2)$. Simultane- ous with the discovery that matching HLA antigens are necessary for optimal success of allogeneic BM transplantation (3), clinical results demonstrated the powerful alloimmune reactions against mHAgs. In an HLA-matched hematopoietic stem cell transplantation (HSCT) setting, mHAg disparities between recipient and donor can lead to graft-versus-host disease (GVHD) (4) or graft rejection (5). Aside from these detrimental effects, the mHAg-induced alloimmune response also causes the curative graft-versus-leukemia (GVL) effect. Since mHAg-specific $T$ cells are involved in both GVHD and GVL, dissecting the role of these cells in the immunobiology of GVHD and GVL has proven challenging. The first indication that led us to propose the use of mHAgs as immunotherapeutic tools in HSCT (6) was provided by the results of in vitro studies showing differential modes of recognition of various cell types by mHAgspecific CTLs, i.e., ubiquitous or hemato- 
Table 1

Immunotherapeutic mHAgs

\begin{tabular}{|c|c|c|c|c|c|c|c|}
\hline $\mathrm{mHAg}$ & $\begin{array}{c}\mathrm{mHAg} \\
\text { disparity }^{\mathrm{A}}\end{array}$ & $\begin{array}{l}\text { HLA-restriction } \\
\text { molecule }\end{array}$ & $\begin{array}{c}\mathrm{HLA} \\
\text { frequency }\end{array}$ & $\begin{array}{c}\text { Overall } \\
\text { applicability }\end{array}$ & $\begin{array}{c}\mathrm{mHAg} \\
\text { gene }\end{array}$ & mHAg tissue distribution & Reference \\
\hline $\mathrm{HA}-1$ & $24 \%$ & HLA-A2 & $43 \%$ & $10.6 \%$ & $H A-1$ & $\begin{array}{l}\text { Hematopoietic cells, myeloid and lymphoid } \\
\text { leukemic cells }\end{array}$ & $(29)$ \\
\hline $\mathrm{HA}-2$ & $4 \%$ & HLA-A2 & $43 \%$ & $1.7 \%$ & Myosin 1G & $\begin{array}{l}\text { Hematopoietic cells, myeloid and lymphoid } \\
\text { leukemic cells }\end{array}$ & $(15)$ \\
\hline $\mathrm{HB}-1 \mathrm{H} / \mathrm{YC}$ & $6 \% / 24 \%$ & HLA-B44 & $12 \%$ & $0.7 \% / 2.9 \%$ & Unknown & B cell ALL & $(16)$ \\
\hline ACC-1 & $17 \%$ & HLA-A24 & $34 \%$ & $5.8 \%$ & $B C L 2 A 1$ & $\begin{array}{l}\text { Hematopoietic cells, myeloid and lymphoid } \\
\text { leukemic cells }\end{array}$ & (27) \\
\hline ACC-2 & $17 \%$ & HLA-B44 & $12 \%$ & $2.7 \%$ & $B C L 2 A 1$ & $\begin{array}{l}\text { Hematopoietic cells, myeloid and lymphoid } \\
\text { leukemic cells }\end{array}$ & $(27)$ \\
\hline UGT2B17 & $11 \%$ & HLA-A29 & $5 \%$ & $0.6 \%$ & UGT2B17 & DCs, B cells & $(17)$ \\
\hline $\mathrm{LRH}-1$ & $13 \% D$ & HLA-B7 & $11 \%$ & $1.4 \%$ & $P 2 \times 5$ & $\begin{array}{l}\text { T cells, B cells, NK cells, myeloid leukemic } \\
\text { progenitor cells }\end{array}$ & (28) \\
\hline $\mathrm{B} 8 / \mathrm{H}-\mathrm{Y}$ & $25 \%$ & HLA-B8 & $8 \%$ & $2.0 \%$ & UTY & Hematopoietic cells & $(30)$ \\
\hline
\end{tabular}

ADisparity within the transplant pairs with the correct HLA allele. Calculations were based on the reported allele frequencies under the assumption of an HLA-matched unrelated donor. BPhenotype frequencies were calculated based on global allele frequencies reported in dbMHC (http://www.ncbi.nlm. nih.gov/mhc). CHB-1 can be recognized bidirectionally. Data represent, respectively, the disparity for $\mathrm{HB}-1^{\mathrm{H}}$ and $\mathrm{HB}-1^{\mathrm{Y}}$ as positive alleles. DIt has not been reported whether the disparity rate for $\mathrm{LRH}-1$ was determined in $\mathrm{HLA}$-matched sibling pairs or in HLA-matched unrelated donor/recipient pairs. ALL, acute lymphocytic leukemia.

poietic system-restricted (7). mHAgs are ubiquitously expressed, includeing on $t$ fibroblasts, melanocytes, and keratinocytes; cell types present within organs affected by GVHD. The CTLs directed to the ubiquitous mHAgs are therefore particularly relevant to the development of GVHD. In addition to in vitro cellular analyses, an in situ readout was performed to analyze the postulated differential in vivo effects of mHAgs. For this, an ex vivo in situ skin explant assay was used, wherein skin sections were incubated with CTLs specific for a ubiquitously expressed mHAg, H-Y, or for the hematopoietic system-restricted mHAgs HA-1 and HA-2 (8). CTLs specific for the H-Y mHAg induced severe graft-versus-host reactions of grades III-IV. CTLs specific for HA-1 and HA-2 induced no or weak graft-versus-host reactions.

mHAgs with tissue expression limited to cells of the hematopoietic system are especially relevant to GVL activity. CTLs specific for hematopoietic system-restricted mHAgs are capable of lysing leukemic cells in vitro (9) and in vivo in a translational mouse model (E. Goulmy et al., unpublished observations). In the clinical setting of HSCT, complete hematological responses and conversion from mixed to complete donor chimerism after donor lymphocyte infusion (DLI) for the treatment of chronic myeloid leukemia and multiple myeloma are associated with a rapid increase in the numbers of functional HA-1- and HA-2specific $\mathrm{T}$ cells in peripheral blood (10).
These data strongly suggest that donor $\mathrm{T}$ cells specific for hematopoietic systemrestricted mHAgs expressed on recipient cells can be involved in the induction and/ or maintenance of remission of hematological malignancies after HSCT.

The accumulated in vitro and in vivo data underline the proposition that mHAgs could be used to induce the curative effect of HSCT. It is noteworthy that this application is not restricted to hematological malignancies but extends to solid tumors as well (11). Protocols have been established for the in vitro generation of donorderived HA-1- or HA-2-specific CTLs to treat recurrence of the original disease after HLA-matched HA-1-mismatched and/or HLA-matched HA-2-mismatched HSCT (12). A potentially efficient strategy is vaccination of patients by boosting the donor GVL response at appropriate times after HSCT with minor histocompatibility peptides. Currently, an HA-1/HA-2 phase I/II vaccination trial for HLA-A2/HA-1positive and/or HLA-A2/HA-2 -positive patients with advanced hematological malignancies receiving HLA-matched HA-1-mismatched and/or HLA-matched HA-2-mismatched HSCT is ongoing. It is hoped that this approach will elicit allogeneic responses against mHAgs HA-1 or HA-2 and will result in an anti-leukemic effect (Koen van Besien, University of Chicago, Chicago, Illinois, USA, personal communication). We expect that the results of this trial will serve as proof-of-principle and will lay the basis for second-generation vaccination trials.

\section{Current possibilities for mHAg- specific immunotherapy}

The immunotherapeutic potential of celland/or tissue-restricted mHAgs demands serious searches for new mHAgs. Information on their phenotypic frequency, tissue distribution, functional membrane expression, and epidemiology is indispensable. The disparity rate of the mHAg between 2 unrelated individuals combined with the allele frequency of the HLA restriction molecule determines its overall applicability. With an overall applicability rate of $10.6 \%$, HA- 1 is currently the most interesting candidate for mHAg-based immunotherapy. So far, only 6 other mHAgs with hematopoietic system-specific tissue distribution have been described; 5 are encoded by autosomal genes, and 1 is encoded on the Y chromosome (Table 1; reviewed in ref. 13). Despite inclusion of these mHAgs, the potential number of patients that could be treated remains low due to the phenotypic frequencies of the mHAgs and the HLA restriction molecule.

\section{mHAg identification systems}

Various biochemical and molecular approaches have been used to characterize mHAgs. The classical way to identify human mHAgs is elution of peptides from the relevant HLA molecules. The strength of this approach is that the identified 
peptide is by definition present on the cell surface $(14,15)$. The recent determination of the complete human genome sequence has facilitated identification of the gene encoding the relevant peptide. The drawback of the classical approach is the need for highly specialized equipment and personnel. Alternatively, cDNA library screening has successfully been executed for the identification of antigenic minor histocompatibility peptides. Although this technique can be applied for identifying autosomal mHAgs $(16,17)$, it is particularly powerful for identifying $\mathrm{H}-\mathrm{Y}$ epitopes, for which there are only a limited number of candidate genes (18-21).

Another possibility for the chemical identification of human mHAgs was put forward by Gubarev et al. in 1996 (22); these authors suggested application of genetic linkage analysis to identify mHAg loci. The method uses EBV-transformed lymphoblastoid cell lines from large families (for example, from the Centre d'Etude du Polymorphism Humain panel). The families studied consist of 3 generations, and all individuals have been typed for 3,000-10,000 genetic markers $(23,24)$. This approach led to the localization of mHAgs on chromosomes 22 (22) and 11 (25). At that time, these mHAg loci could not be further refined, leaving the biochemical structure of the epitopes unsolved. The first indications that this approach could indeed lead to the molecular identification of minor histocompatibility peptides were provided by a retrospective study on the HA-8 antigen (26). Combining the genetic linkage data with HLA-binding prediction tools on nonsynonymous single nucleotide polymorphism-containing DNA sequences yielded an epitope that matched the eluted one. Subsequently, this methodology was utilized for the molecular identification of 2 BCL2A1-encoded mHAg T cell epitopes, i.e. ACC-1 and ACC-2 (27). The genetic linkage analyses combined with the $\mathrm{T}$ cell reactivities specific for mHAgs in question resulted in 46 candidate genes. Further identification was facilitated by the fact that ACC-1- and ACC-2-specific T cell clones only recognize cells of the hematopoietic system. The only gene that was reported by databases to match the expression pattern was $B C L 2 A 1$. Peptide reconstitution assays finally resolved the biochemical identity of the ACC- 1 and ACC- 2 mHAg $\mathrm{T}$ cell epitopes.

In this issue of the JCI, de Rijke et al. describe an identical approach that they used in order to identify lymphoid-restrict- ed histocompatibility antigen-1 (LRH-1) (28). To circumvent the problem that tissue distribution data in the various databases might be incomplete or incorrect, real-time PCR analysis of candidate genes was performed. This additional selection procedure appeared to be crucial for identifying the correct gene. The results clearly show that molecular identification via genetic linkage analyses can successfully be executed for mHAgs with a limited tissue distribution. Genetic linkage identification of minor histocompatibility epitopes with a broad expression pattern, such as the GVHD-associated mHAgs, might turn out to be more difficult. For the identification of leukemia-specific mHAgs and mHAgs that are not expressed by EBV-transformed lymphoblastoid cell lines, this approach is not applicable.

\section{Implications of LRH-1 use for adoptive immunotherapy}

The novel mHAg LRH-1 is encoded by the hematopoietic system-specific P $2 X 5$ gene, which has interesting properties with respect to HSCT-based immunotherapy of hematological malignancies. First, $P 2 X 5$ transcripts were only detected in lymphoid cells and myeloid leukemia progenitor cells. De Rijke et al. analyzed the presence of LRH-1-specific T cells following HSCT and DLI in a patient with chronic myeloid leukemia (28). A massive rise in the number of LRH-1-specific CTLs coincided with a reduction in the number of Bcr-Abl-positive cells, indicating a potential role for these $\mathrm{T}$ cells in the clinical response to LRH-1-expressing CD34+ leukemia progenitor cells.

In addition to their hematopoietic system-restricted expression, the mHAg phenotypic frequency and frequency of its HLA restriction molecule represent significant characteristics of mHAgs that make them suitable for use in adoptive immunotherapy. Within the transplant pairs with the correct HLA allele, de Rijke et al. report a $13 \%$ disparity, a situation where the transplant donor is negative, and the transplant recipient is positive, for the LHR-1 antigen (28). The LRH-1 mHAg is presented to the immune system by the HLA-B7 molecule. With a phenotype frequency in the range of $10-25 \%$, HLA-B7 is among one of the more frequent HLA alleles (according to a search of the dbMHC; http://www.ncbi.nlm.nih. gov/mhc). The combined LHR-1 and HLAB7 phenotypic frequency clearly positions LHR-1 on the list of candidate mHAgs suitable for immunotherapy of hematological malignancies. More importantly, the applicability of $P 2 X 5$ gene products might exceed that of the LRH-1 epitope. De Rijke et al. found that the P2X5 gene of the HSCT recipient contained a deletion of a single nucleotide, resulting in a frameshift. To our knowledge, generation of an mHAg via nucleotide deletion/insertion has not been described before and presents interesting opportunities to further exploit the $P 2 X 5$ gene product as a source for mHAgs to be used for immunotherapy. HSCT donor T cells might be able to recognize peptides derived from the recipient's $P 2 X 5$ gene product fragment following the frameshift. Further investigations using the reverse immunology strategy on this part of the protein might yield new mHAgs in the context of the frequent HLA class I alleles. Moreover, it would be of interest to evaluate whether the C-terminal part of the P2X5 gene product might contain HLA class II-restricted mHAgs. Evidently, CD4 $\mathrm{T}$ cell help during the in vitro and/or in vivo generation of LRH-1-specific CTLs is crucial. Identification of a combination of functionally different types of mHAgs will definitely further support successful mHAg-based immunotherapy.

Address correspondence to: Els Goulmy, Leiden University Medical Center, Department of Immunohematology and Blood Transfusion, PO Box 9600, 2300 RC Leiden, The Netherlands. Phone: 31-715261966; Fax: 31-71-5216751; E-mail: e.a.j.m.goulmy@lumc.nl.

1. Snell, G.D. 1948. Methods for the study of histocompatibility genes. J. Genet. 49:87-103.

2. Counce, C., Smith, P., Barter, R., and Snell, G.D. 1956. Strong and weak histocompatibility fine differences in mice and their role in the rejection of homografts of tumors and skin. Ann. Surg. 144:198-204.

3. Thomas, E., et al. 1975. Bone-marrow transplantation. N. Engl. J. Med. 292:832-902.

4. Goulmy, E., Gratama, J.W., Blokland, E., Zwaan, F.E., and van Rood, J.J. 1983. A minor transplantation antigen detected by MHC-restricted cytotoxic $\mathrm{T}$ lymphocytes during graft-versus-host disease. Nature. 302:159-161.

5. Goulmy, E., Termijtelen, A., Bradley, B.A., and van Rood, J.J. 1976. Alloimmunity to human H-Y [letter]. Lancet. 2:1206.

6. Goulmy, E. 1997. Human minor histocompatibility antigens: new concepts for marrow transplantation and adoptive immunotherapy. Immunol. Rev. 157:125-140.

7. de Bueger, M.M., Bakker, A., van Rood, J.J., Van der Woude, F., and Goulmy, E. 1992. Tissue distribution of human minor histocompatibility antigens. Ubiquitous versus restricted tissue distribution indicates heterogeneity among human cytotoxic $\mathrm{T}$ lymphocyte-defined non-MHC antigens. J. Immunol. 
149:1788-1794.

8. Dickinson, A.M., et al. 2002. In situ dissection of the graft-versus-host activities of cytotoxic T cells specific for minor histocompatibility antigens. Nat Med. 8:410-414.

9. van der Harst, D., et al. 1994. Recognition of minor histocompatibility antigens on lymphocytic and myeloid leukemic cells by cytotoxic T-cell clones. Blood. 83:1060-1066.

10. Marijt, W.A., et al. 2003. Hematopoiesis-restricted minor histocompatibility antigens HA-1- or HA-2 specific $\mathrm{T}$ cells can induce complete remissions of relapsed leukemia. Proc. Natl. Acad. Sci. U. S. A. 100:2742-2747.

11. Goulmy, E. 2004. Minor histocompatibility antigens: allo target molecules for tumor-specific immunotherapy. Cancer J. 10:1-7.

12. Mutis, T., et al. 1999. Feasibility of immunotherapy of relapsed leukemia with ex vivo- generated cytotoxic T lymphocytes specific for hematopoietic system-restricted minor histocompatibility antigens. Blood. 93:2336-2341.

13. Spierings, E., Wieles, B., and Goulmy, E. 2004 Minor histocompatibility antigens - big in tumour therapy. Trends Immunol. 25:56-60.

14. Rotzschke, O., Falk, K., Wallny, H.J., Faath, S., and Rammensee, H.G. 1990. Characterization of naturally occurring minor histocompatibility peptides including H-4 and H-Y. Science. 249:283-287.

15. den Haan, J.M., et al. 1995. Identification of a graft versus host disease-associated human minor histocompatibility antigen. Science. 268:1476-1480.

16. Dolstra, H., et al. 1999. A human minor histocompatibility antigen specific for B cell acute lymphoblastic leukemia. J. Exp. Med. 189:301-308.

17. Murata, M., Warren, E.H., and Riddell, S.R. 2003. A human minor histocompatibility antigen resulting from differential expression due to a gene deletion. J. Exp. Med. 197:1279-1289.

18. Vogt, M.H., de Paus, R.A., Voogt, P.J., Willemze, R., and Falkenburg, J.H. 2000. DFFRY codes for a new human male-specific minor transplantation antigen involved in bone marrow graft rejection. Blood. 95:1100-1105

19. Vogt, M.H., et al. 2000. UTY gene codes for an HLA-B60-restricted human male-specific minor histocompatibility antigen involved in stem cell graft rejection: characterization of the critical polymorphic amino acid residues for $\mathrm{T}$-cell recognition. Blood. 96:3126-3132.

20. Vogt, M.H., et al. 2002. The DBY gene codes for an HLA-DQ5 restricted human male specific minor histocompatibility antigen involved in GvHD. Blood. 99:3027-3032.

21. Spierings, E., et al. 2003. Identification of HLA class II-restricted H-Y-specific T-helper epitope evoking CD4+ T-helper cells in H-Y-mismatched transplantation. Lancet. 362:610-615.

22. Gubarev, M.I., et al. 1996. Localization to chromosome 22 of a gene encoding a human minor histo- compatibility antigen. J. Immunol. 157:5448-5454. 23. Dausset, J., et al. 1990. Centre d'etude du polymorphisme humain $(\mathrm{CEPH})$ : collaborative genetic mapping of the human genome. Genomics. 6:575-577.

24. Murray, J.C., et al. 1994. A comprehensive human linkage map with centimorgan density. Cooperative Human Linkage Center (CHLC). Science. 265:2049-2054.

25. Gubarev, M.I., et al. 1998. Localization to chromosome 11 of a gene encoding a human minor histocompatibility antigen. Exp. Hematol. 26:976-981.

26. Warren, E.H., et al. 2002. Feasibility of using genetic linkage analysis to identify the genes encoding $\mathrm{T}$ cell-defined minor histocompatibility antigens. Tissue Antigens. 59:293-303.

27. Akatsuka, Y., et al. 2003. Identification of a polymorphic gene, BCL2A1, encoding two novel hematopoietic lineage-specific minor histocompatibility antigens. J. Exp. Med. 197:1489-1500.

28. de Rijke, B., et al. 2005. A frameshift polymorphism in $P 2 X 5$ elicits an allogeneic cytotoxic T lymphocyte response associated with remission of chronic myeloid leukemia. J. Clin. Invest. 115:3506-3516. doi:10.1172/JCI24832.

29. den Haan, J.M., et al. 1998. The minor histocompatibility antigen HA-1: a diallelic gene with a single amino acid polymorphism. Science. 279:1054-1057.

30. Warren, E.H., et al. 2000. The human UTY gene encodes a novel HLA-B8-restricted H-Y antigen. J. Immunol. 164:2807-2814.

\title{
Flushing out the role of GPR109A (HM74A) in the clinical efficacy of nicotinic acid
}

\author{
Nicholas B. Pike
}

Atherosclerosis Department, GlaxoSmithKline, Stevenage, United Kingdom.

\begin{abstract}
The recent discovery of the $G_{i}$ protein-coupled receptor GPR109A (HM74A in humans; PUMA-G in mice) as a receptor for nicotinic acid has provided the opportunity to gain greater understanding of the underlying biology contributing to the clinical efficacy (increases in HDL, decreases in VLDL, LDL, and triglycerides) and the characteristic side-effect profile of nicotinic acid. GPR109A has been proven to be the molecular target for the actions of nicotinic acid on adipose tissue, and in this issue of the JCI, Benyó et al. have confirmed the involvement of GPR109A in the nicotinic acid-induced flushing response, a common side effect (see the related article beginning on page 3634). The involvement of GPR109A in both the desirable and undesirable clinical actions of nicotinic acid raises interesting questions regarding the function of this receptor.
\end{abstract}

The observation that nicotinic acid can modify lipoprotein profiles in humans was first made in the 1950s. Subsequent clinical experience has demonstrated that nicotinic acid produces a very beneficial modification of multiple cardiovascular risk factors. As a

Nonstandard abbreviations used: $\mathrm{PGD}_{2}$, prostaglandin $\mathrm{D}_{2} ; \mathrm{PGE}_{2}$, prostaglandin $\mathrm{E}_{2} ;$ PUMA-G, protein upregulated in macrophages by IFN- $\gamma$.

Conflict of interest: The author has declared that no conflict of interest exists.

Citation for this article: J. Clin. Invest. 115:3400-3403 (2005). doi:10.1172/JCI27160. monotherapy, nicotinic acid is still the most effective therapy for elevating HDL levels while decreasing VLDL and LDL levels as well as other cardiovascular risk factors, which results in a reduction in mortality (1) (Figure 1). In addition to its highly desirable profile of cardiovascular risk factor modulation, nicotinic acid has been shown to produce enhanced therapeutic effects when given in combination with other lipid-lowering drugs (e.g., statins and bile acid resins) (2-3). The past 50 years of nicotinic acid usage has been recently reviewed by Carlson (4).

\section{Identification and function of $G_{\text {i }}$ protein-coupled receptors for nicotinic acid}

In 2003, several groups published studies showing that the orphan receptor GPR109A is activated by nicotinic acid at concentrations consistent with the exposure achieved following therapeutic doses (5-7). Furthermore, additional compounds with a clinical profile similar to that of nicotinic acid (e.g., acipimox and acifran) were also confirmed as full agonists of GPR109A. Importantly, nicotinamide, which does not alter lipoprotein profiles but shares the vitamin-like properties of nicotinic acid, has virtually no GPR109A agonist activity. This pharmacological profile strongly suggests that GPR109A is a molecular target involved in the clinical efficacy of nicotinic acid and therefore offers a potential focus to explore the biological processes involved in the highly desirable therapeutic profile achieved following chronic treatment with this drug (8-9).

The best-described action of nicotinic acid is the inhibition of adipocyte lipolysis. 